UCRL-JC-122108

PREPRINT

CONF-950706-18

\title{
Recent Results from the Super EBIT
}

R. E. Marrs

This paper was prepared for submittal to the The Physics of Electronic and Atomic Collisions

XIX International Conference

Whistler, Canada

July 26-August 1, 1995

September 15, 1995

This is a preprint of a paper intended forpublication in a journal or proceedings. Since changes may be made before publication, this preprint is made available with the understanding that it will not be cited or reproduced without the permission of the author.

\section{OSTI}

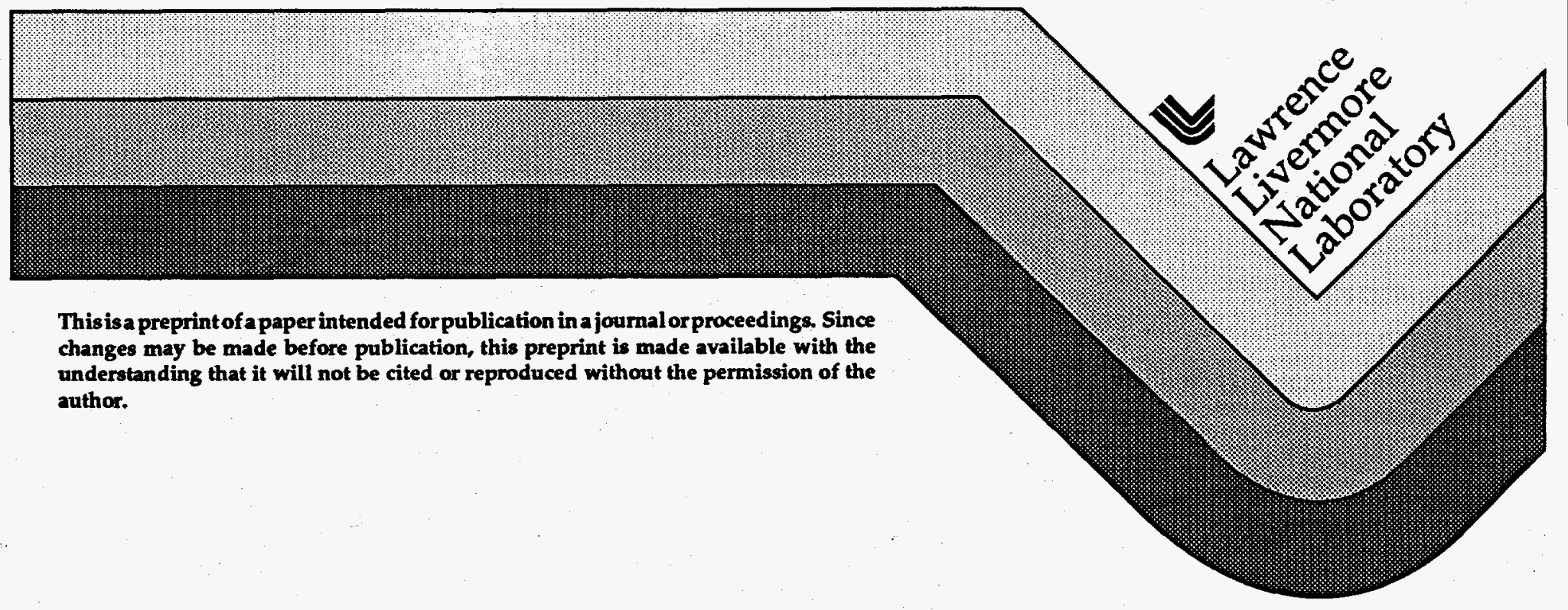




\section{DISCLAIMER}

This document was prepared as an account of work sponsored by an agency of the United States Government. Neither the United States Government nor the University of California nor any of their employees, makes any warranty, express or implied, or assumes any legal liability or responsibility. for the accuracy, completeness, or usefulness of any information, apparatus, product, or process disclosed, or represents that its use would not infringe privately owned rights. Reference herein to any specific commercial products, process, or service by trade name, trademark, manufacturer, or otherwise, does not necessarily constitute or imply its endorsement, recommendation, or favoring by the United States Government or the University of California. The views and opinions of authors expressed herein do not necessarily state or reflect those of the United States Government or the University of California, and shall not be used for advertising or product endorsement purposes. 


\section{DISCLAIMER}

Portions of this document may be illegible in electronic image products. Images are produced from the best available original document. 


\title{
Recent Results from the Super EBIT
}

\author{
R. E. Marrs \\ Lawrence Livermore National Laboratory \\ Livermore, CA 94550
}

\begin{abstract}
The Super EBIT device at LLNL can produce and trap any highly charged ion at rest in the laboratory, including bare $\mathrm{U}^{92+}$ ions. Recently, the ionization cross sections for high- $Z$ hydrogenlike ions have been measured for the first time, and measurements of the L-shell ionization cross sections for uranium ions are in progress. The two-electron contributions to the ground state energies of heliumlike ions have been directly measured using a novel technique, and spectra of $2 \mathrm{~s}-2 \mathrm{p}$ transitions in highly ionized thorium and uranium have been used to test QED corrections to the energy levels of few electron high-Z ions. A new capability for the study of rare isotopes has been demonstrated. Ion cooling has been used to reduce the thermal broadening of $\mathrm{x}$-ray emission lines to the point where natural line widths can be observed in some cases.
\end{abstract}

\section{INTRODUCTION}

The super electron beam ion trap (Super EBIT) is a device for producing and trapping the few-electron ions of heavy elements for studies of atomic structure and electron-ion collisions. It is called "Super" because of the high $(\sim 200 \mathrm{keV})$ electron energy. Any highly charged ion can be produced in a Super EBIT, including bare $\mathrm{U}^{92+}$ [1]. Such highly charged ions provide an opportunity to understand the contributions of relativity and QED in atomic structure and interactions. For example, QED energy corrections increase as $Z^{4}$, so they are most visible and most important at high atomic number.

In an EBIT, atomic physics information is derived from the $\mathrm{x}$-ray emission spectra of trapped ions colliding with beam electrons. The ions are confined radially by the space charge potential of the electron beam. In the axial direction the ions are confined by bias voltages applied to three drift tubes. The electron beam is compressed to high density by the magnetic field from a split pair of superconducting coils; the magnetic field has little effect on the ion motion. $\mathrm{X}$ rays are observed at 90 degrees to the electron beam through the gap between the coils as indicated in Fig. 1. A more detailed description of the LLNL Super EBIT may be found elsewhere [2]. 


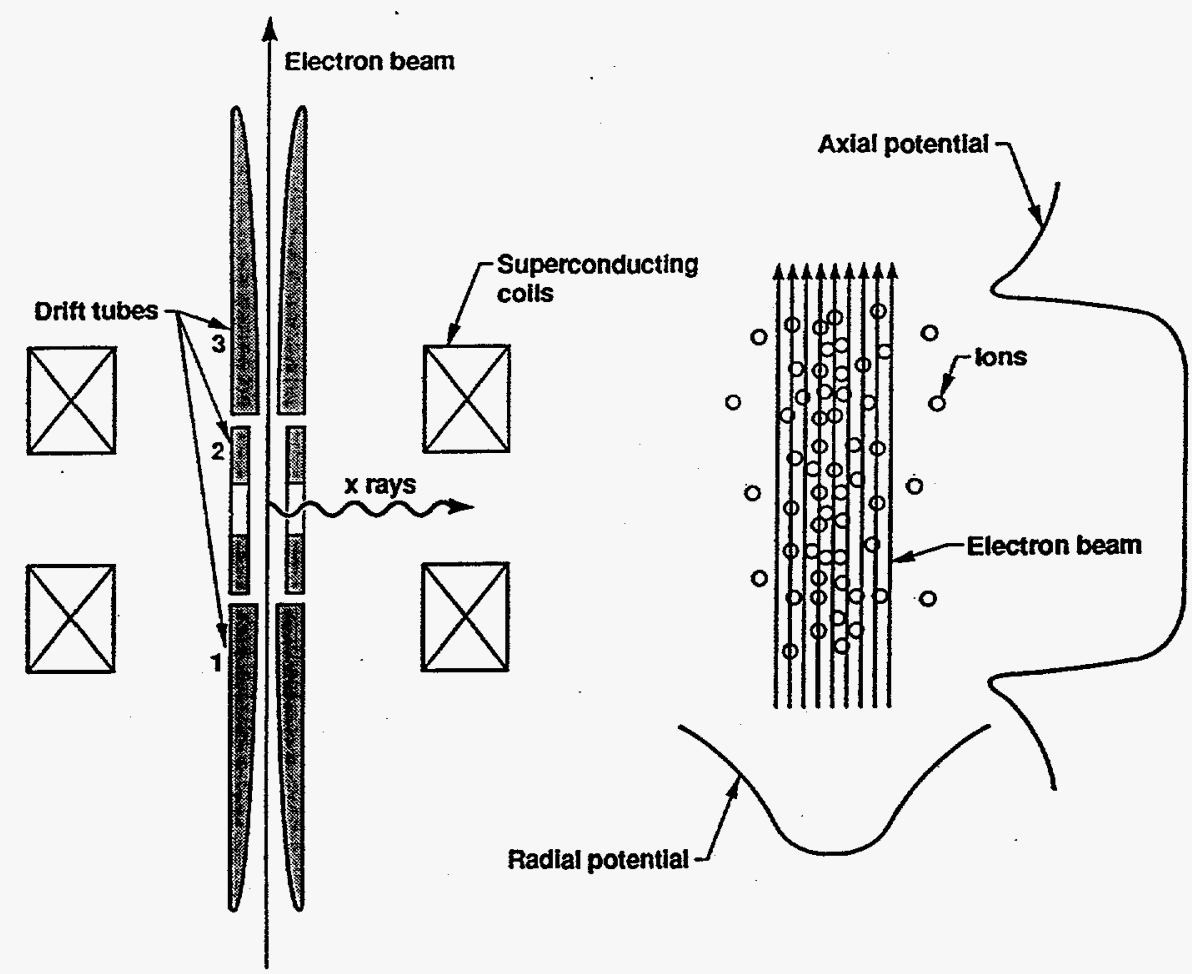

FIGURE 1. Key features of the LLNL Super EBIT. Left: Scale drawing of the trap electrodes and magnet coils. Right: Schematic enlargement of the trapping potentials. Ions are confined in drift tube number 2.

Recent Super EBIT experiments at LLNL span two orders of magnitude in $\mathrm{x}$-ray energy and address several different areas of highly-charged-ion physics. Most of the recent results at high electron energy are summarized in what follows. The results of many other EBIT experiments at lower electron energy can be found in the literature.

\section{PRODUCTION OF BARE U $^{92+}$ IONS}

Bare $U^{92+}$ is sometimes called the ultimate ion, since it is the highest ionization stage of the heaviest naturally occurring element, as well as the most difficult ion to produce. The threshold for producing $\mathrm{U}^{92+}$ by electron impact ionization of the preceding hydrogenlike ionization stage is approximately 130 $\mathrm{keV}$, within the range of a Super EBIT. Trapped bare uranium ions were first produced in the LLNL Super EBIT, operating at its maximum energy of $200 \mathrm{keV}$ [1]. Unfortunately, at this energy, the radiative recombination cross section for destroying $\mathrm{U}^{92+}$ is roughly 40 times larger than the ionization cross section for producing it, so only a small fraction of the trapped uranium ions can be in the fully 
stripped charge state. However, as discussed below, enough bare uranium is produced to use its abundance as a signal for the measurement of the ionization cross section that produces it. The other (few electron) ionization stages of uranium can be produced in sufficient abundance for high resolution spectroscopy of their characteristic X-ray lines. Table I lists the observed uranium ionization balance as derived from radiative recombination spectra such as the one shown in Fig. 2.

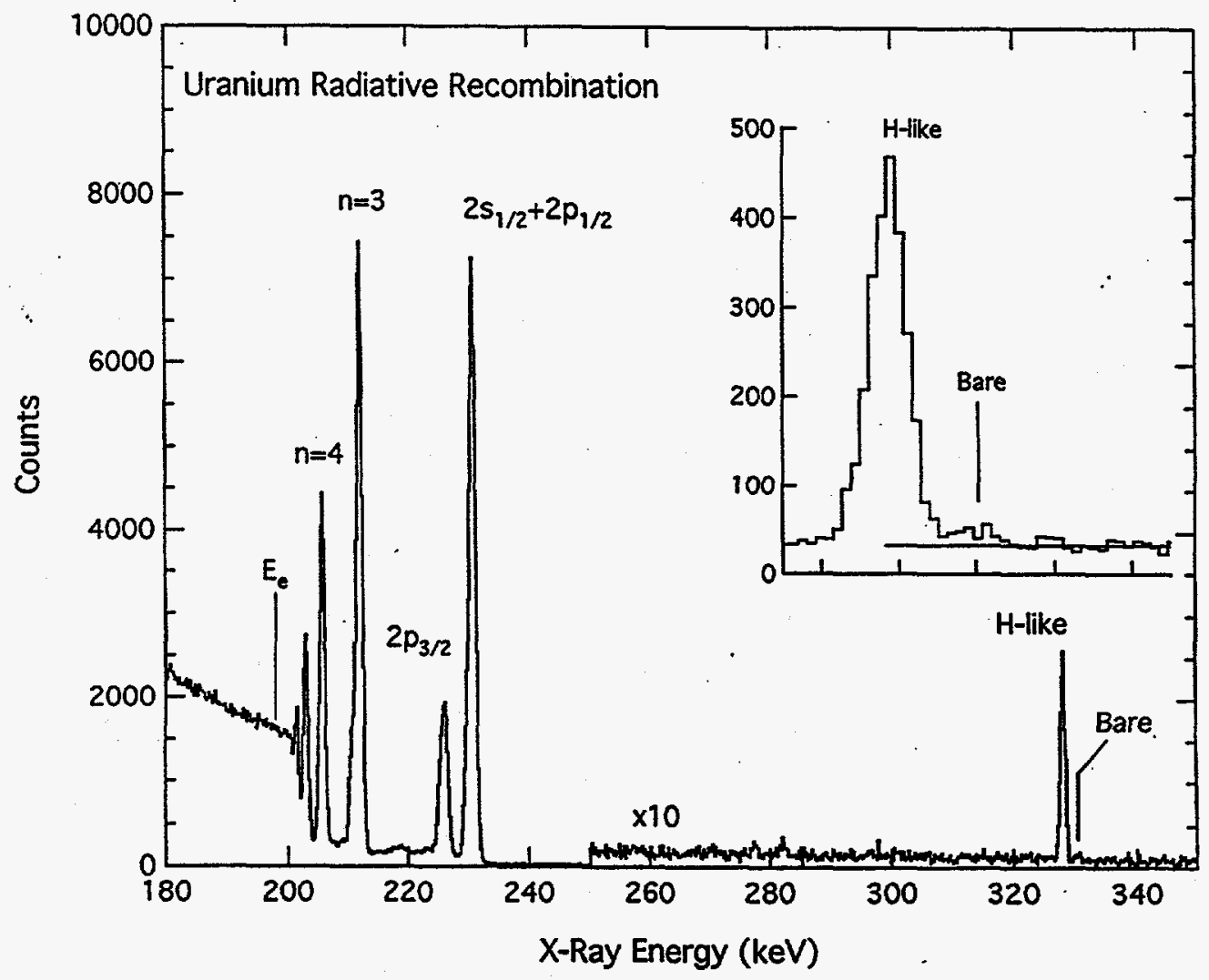

FIGURE 2. Uranium radiative recombination $x$-ray spectrum at 198-keV electron energy observed in a $40-\mathrm{cm}^{3}$ coaxial germanium detector. The inset shows the $n=1$ feature from a second $\left(90-\mathrm{cm}^{3}\right)$ detector.

\section{ELECTRON-ION COLLISION CROSS SECTIONS}

Every $x$ ray emitted by the ions trapped in an EBIT is associated with an electron-ion collision. The $x$-ray spectra, in combination with the ability to vary the electron energy that produces them, enable the study of almost every type of electron-ion collision process. Moreover, the cross section for one collision 
TABLE I. The observed equilibrium ionization balance in the LLNL Super EBIT for uranium at approximately 200-keV electron beam energy.

\begin{tabular}{ll}
$\begin{array}{ll}\text { lonization } \\
\text { stage }\end{array}$ & Abundance (\%) \\
\hline Bare & $0.02 \%$ \\
H-like & $1.0 \%$ \\
He-like & $17 \%$ \\
Li-like & $34 \%$ \\
Be-like & $31 \%$ \\
B-like & $15 \%$ \\
C-like & $3 \%$ \\
\hline
\end{tabular}

process, radiative recombination, is well known [3], so it can be used to normalize measurements of the cross sections for other processes such as ionization, excitation, or dielectronic recombination of the same target ions. This avoids the errors associated with an absolute cross section measurement. Almost all EBIT cross section measurements have been normalized to radiative recombination, which produces easily observable $\mathrm{x}$-ray lines for every target ion.

Recent Super EBIT experiments at LLNL succeeded in making the first direct measurement of the ionization cross section for hydrogenlike uranium, as well as several other hydrogenlike high- $Z$ ions. Measurements of ionization cross sections for the uranium $\mathrm{L}$ shell are in progress. Another electron-ion collision process, dielectronic recombination, has been studied for the first time for highly charged uranium ions with excitation of $\mathrm{K}$-shell electrons.

\section{Ionization}

- The electron impact ionization cross sections for the tightly bound 1s electrons of high- $Z$ elements are of interest as a test of relativistic interactions in a simple atomic system. Until now, the size of such cross sections could only be obtained indirectly from the stripping of accelerator beams [4]. Although the electron impact ionization of a hydrogenlike or heliumlike ion does not directly involve the emission of an $\mathrm{x}$ ray, we have developed an $\mathrm{x}$-ray technique based on the condition of steady-state ionization balance in Super EBIT and used it to measure the ionization cross section of the hydrogenlike ions of several elements, including uranium [1]. In this technique the bare-to-hydrogenlike abundance ratio, $\mathrm{N}_{\mathrm{Bar}} \mathrm{N}_{\mathrm{H}}$, is determined from the relative intensity of $\mathrm{K}$-shell radiative recombination $\mathrm{x}$-rays. The hydrogenlike ionization cross section is then obtained from the abundance ratio using the relation

$$
\sigma_{\mathrm{H} \rightarrow \text { Bare }}^{\mathrm{ion}}=\frac{\mathrm{N}_{\text {Bare }}}{N_{H}}\left(\sigma_{\text {Bare } \rightarrow \mathrm{H}}^{\mathrm{RR}}\right) .
$$


A small correction for charge-exchange-recombination with neutral background gas is required to complete the measurement.

Cross section data for uranium at $198-\mathrm{keV}$ electron energy are shown in Fig. 2. The X-ray spectrum consists of a series of peaks corresponding to radiative recombination into the open shells of the uranium target ions. The lines at approximately $330-\mathrm{keV}$ are from $\mathrm{K}$-shell capture by bare and hydrogenlike target ions. The electron beam energy spread is known from other measurements to be approximately $100 \mathrm{eV} \mathrm{FWHM}$, so its contribution to the observed peak width is hidden by the $900-\mathrm{eV}$ FWHM detector resolution. The intensity of the weak bare uranium radiative recombination line was obtained from a least-squares fit that fixed its width, position, and shape relative to the hydrogenlike radiative recombination line, and used a background level determined from the spectral region above the peak. Separate results were obtained from each of two detectors and averaged. For hydrogenlike uranium, our measured cross section is $50 \%$ larger than theoretical values available at the time [5], a discrepancy that has since been resolved by improvements to the theory[6,7].

Now that the K-shell ionization cross sections have been determined, we are attempting to apply the same technique to the measurement of ionization cross sections for the $\mathrm{L}$ shell of uranium. We determine the equilibrium ionization balance for a population of uranium ions in charge states from $\mathrm{U}^{89+}$ (lithiumlike) to $\mathrm{U}^{82+}$ (neonlike) from the intensity of radiative recombination $\mathrm{x}$ rays associated with each charge state. However, unlike radiative recombination into the $\mathrm{K}$ shell, for which the $\mathrm{x}$-ray lines corresponding to bare and hydrogenlike target ions are completely resolved in a germanium detector, radiative recombination into the $L$ shell of uranium produces incompletely resolved $x$-ray lines. This is illustrated in Fig. 3, which shows an $\mathrm{x}$-ray spectrum obtained at $60-\mathrm{keV}$ electron energy. The intensity of the radiative recombination $x$-ray lines associated with the different charge states can be determined from least squares fits to the $n=2 j=3 / 2$ and $j=1 / 2$ features using the known positions of the $\mathrm{x}$-ray lines. This provides the ionization balance, which is then used to determine the ionization cross sections in a manner similar that used for the hydrogenlike ions. We have obtained data at 45,60 , and $75 \mathrm{keV}$ electron energy.

\section{Dielectronic Recombination}

In dielectronic recombination, an electron (from the Super EBIT beam) is captured by an ion with the simultaneous excitation of a bound electron, resulting in an excited intermediate state. Decay of the intermediate state by photon (x-ray) emission completes the recombination process. Dielectronic recombination is a resonant process because the incident electron energy must exactly match the energy required for excitation (with electron capture). The cross sections are usually very large, producing an easily observed $\mathrm{x}$-ray signal. Resonances in which 


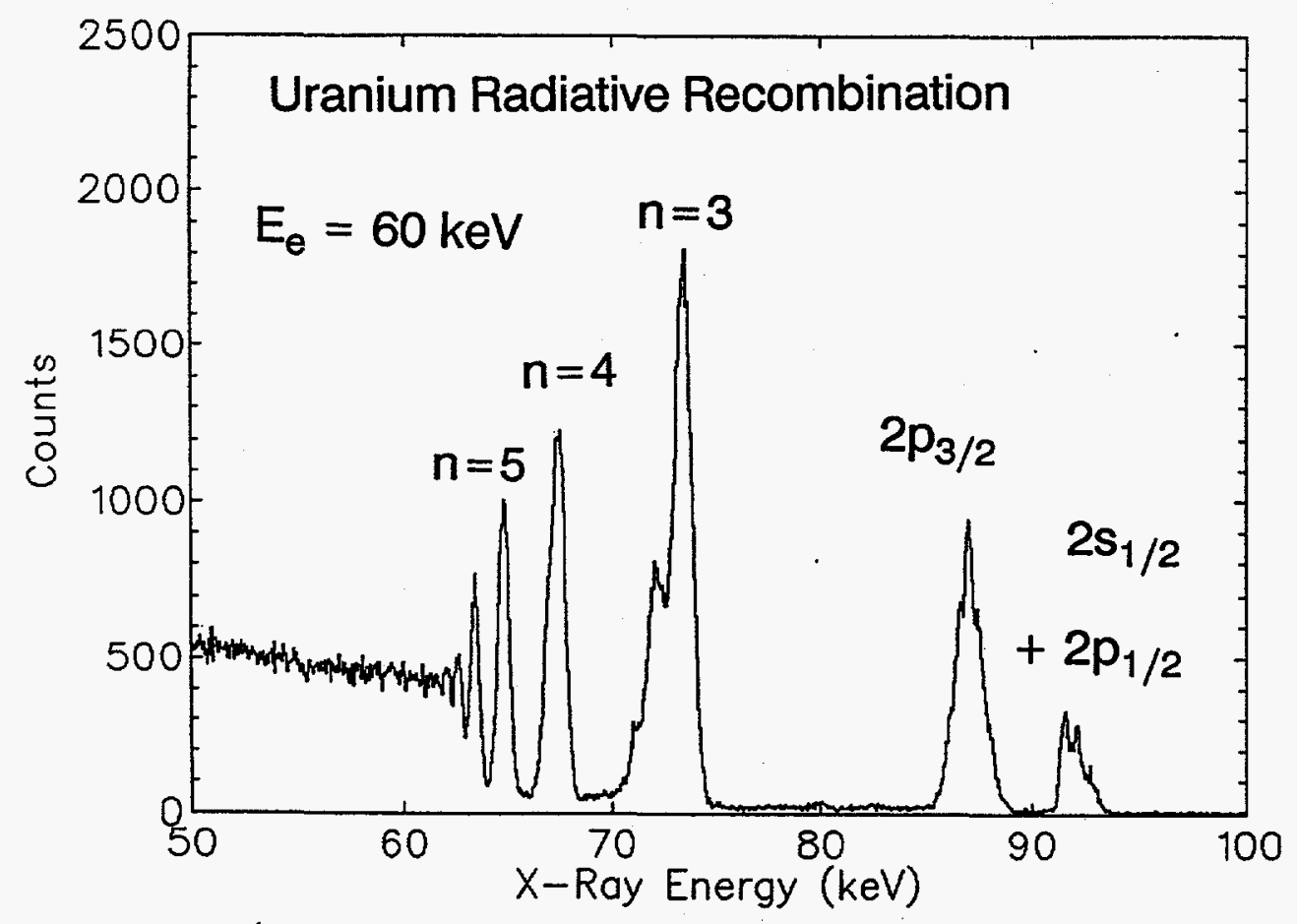

FIGURE 3. Uranium radiative recombination $x$-ray spectrum at $60-k e V$ electron energy observed in a planar germanium detector. The structure in the $n=2 j=3 / 2$ and $j=1 / 2$ features corresponds to the different ionization stages of the target ions.

a K electron is excited to the $\mathrm{L}$ shell and an incident electron is captured into the $\mathrm{L}$ shell are called KLL resonances. The KLL resonances for several heliumlike target ions have been studied in previous EBIT experiments [8,9], but only Super EBIT has sufficient energy to excite the $\mathrm{K}$ electrons in the heaviest elements.

Recently, KLL dielectronic recombination was measured at the LLNL Super EBIT for heliumlike and adjacent ionization stages of uranium, for which the K-L transition energy is roughly $100 \mathrm{keV}$ [10].This experiment was done by scanning the electron beam energy through the resonance region and detecting dielectronic recombination photons in a germanium detector. The most remarkable result of this work is the unexpected discovery of interference between dielectronic and radiative recombination [10].

\section{QED IN HIGH-Z IONS}

Spectroscopic studies of the energy levels of few-electron high- $Z$ ions can be used to understand the effects of quantum electrodynamics (QED) and multielectron corrections in strong Coulomb fields. These effects appear as a difference between the physical energy and the Dirac-Coulomb energy of an atomic state. The one-electron (hydrogenlike) ions are the easiest to treat 
theoretically, and calculations of their energy levels are thought to be highly accurate even for very high $Z$. The heliumlike ions are the simplest multielectron system. However, in contrast to the hydrogenlike ions, there is no exact solution for the structure of high $-Z$ heliumlike ions. The ions corresponding to the range of charge states from lithiumlike to neonlike challenge our understanding of the electron correlation terms that contribute to the energies of these multielectron ions. These issues are addressed by two recent precision $x$-ray experiments with Super EBIT. One experiment measures the high energy $x$ rays emitted in radiative electron capture into the $\mathrm{K}$ shell of bare and hydrogenlike target ions. The other experiment measures the much lower energy $n=2$ to $n=2$ transitions in multielectron ions.

\section{Ground-State Energies In Heliumlike Ions}

Heliumlike ions are more than just hydrogenlike ions with two independent electrons. What makes them different and interesting is the interaction between the two electrons. We have used a novel experimental approach that exploits radiative recombination transitions for a direct measurement of the two-electron contributions to the ground-state energy in heliumlike ions [11]. We measure the difference in the energy of radiative recombination $x$ rays emitted in $K$-shell electron capture by stationary bare and hydrogenlike target ions. This difference is equal to the difference in the ionization potential between the hydrogenlike and heliumlike ion, which is exactly the two-electron contribution to the ground state energy of the heliumlike ion.

It should be noted that the one-electron contributions to the binding energy, such as the finite-nuclear-size correction and the one-electron self energy, cancel out in this type of experiment, which makes such measurements unique. The two-electron contribution to the energy of a heliumlike ion includes, in addition to the dominant Coulomb term, effects from electron correlation, the Breit interaction, screening of the Lamb shift, and higher order radiative corrections. Until now, the precision of available experimental data has not been sufficient to test the theoretical calculation of these various contributions.

The experimental setup at the LLNL Super EBIT is shown in Fig. 4. Energy calibration lines from a radioactive source are mixed with the radiative recombination $x$-ray lines from trapped ions and detected in a coaxial germanium detector. We have obtained data for six different elements: $\mathrm{Ge}(Z=32), \mathrm{Xe}(Z=54)$, $\operatorname{Dy}(Z=66), W(Z=74), \operatorname{Os}(Z=76)$, and $\mathrm{Bi}(Z=83)$. A typical spectrum from the xenon data is shown in Fig. 5. The roughly 100-eV energy spread of the Super EBIT electron beam is much less than the resolution of the $x$-ray detector and does not make a significant contribution to the observed peak width. Because of the rapid decrease in 1 ionization cross sections with increasing atomic number, the abundance of bare target ions is, at present, insufficient to extend these measurements beyond $Z=83$ (see Table I). 


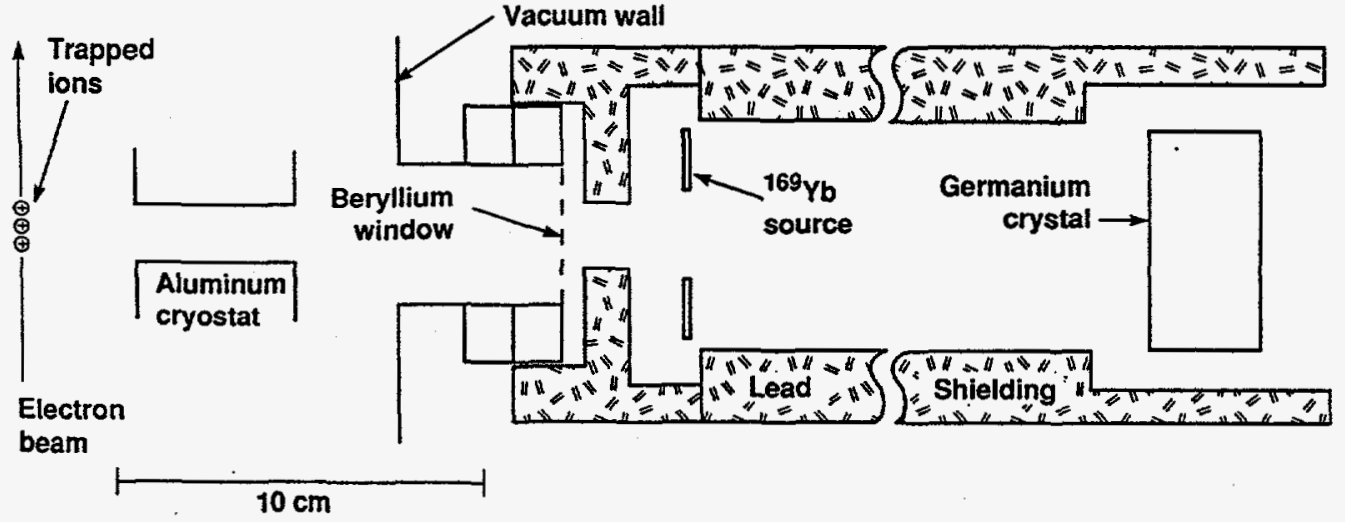

FIGURE 4. Experimental arrangement used for the binding energy measurements. The ${ }^{169} \mathrm{Yb}$ source consists of several removable pieces attached to an annular holder. The detector was located $46 \mathrm{~cm}$ from the trapped ions.

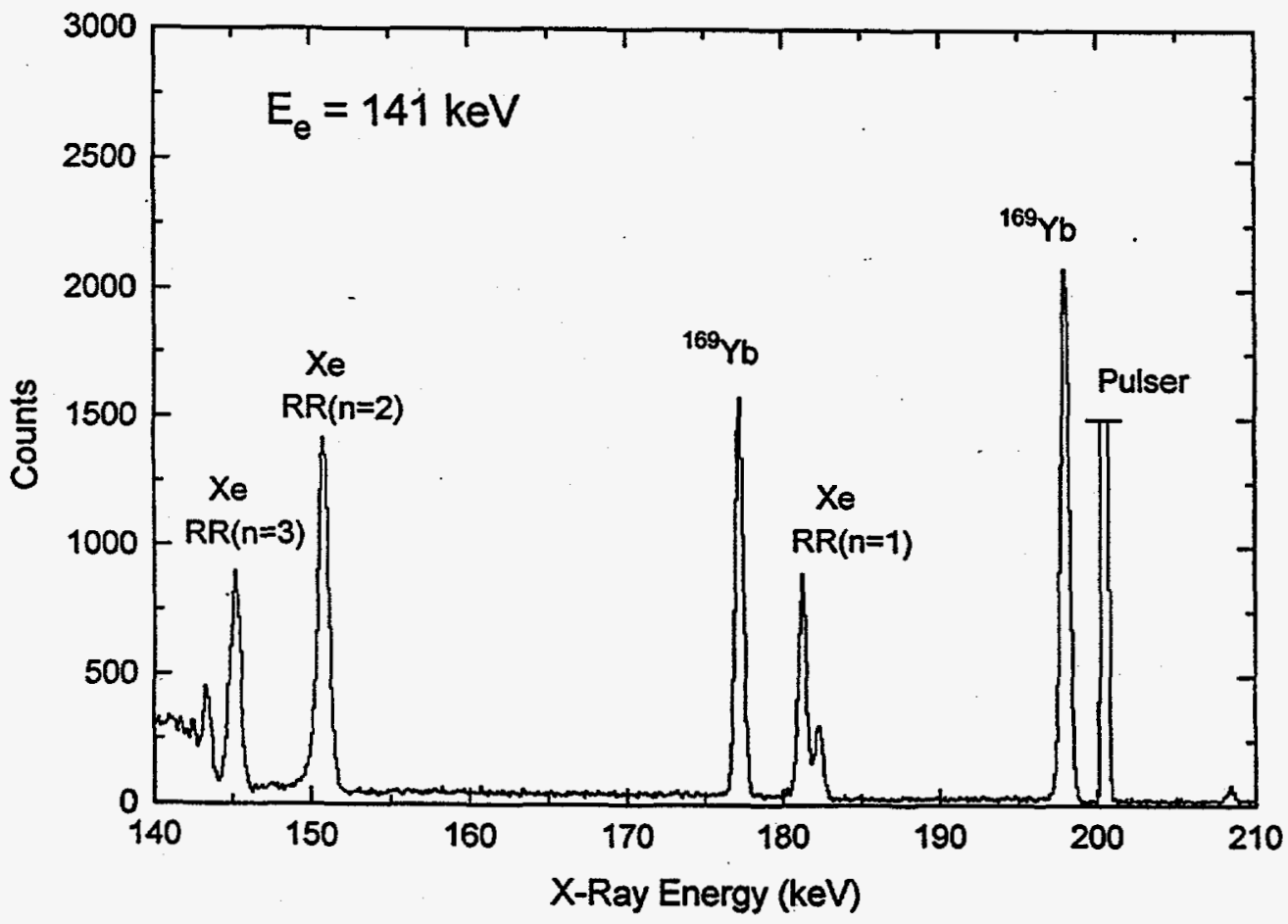

FIGURE 5. Example of spectra used to determine the ionization potential difference for hydrogenlike and heliumlike xenon. The ${ }^{109} \mathrm{Yb}$ lines are from the calibration source. 
TABLE II. The measured two-electron contributions to the ground-state energy of heliumlike ions compared to the relativistic MBPT calculations of Lindgren et al. [11] (in eV

\begin{tabular}{cllll}
\hline$Z$ & $\begin{array}{l}\text { 2nd-Order } \\
\text { Many Body }\end{array}$ & $\begin{array}{l}\text { Two-electron } \\
\text { Lamb Shift }\end{array}$ & $\begin{array}{l}\text { Total } \\
\text { Theory }\end{array}$ & Experiment \\
\hline $\mathrm{Ge}(32)$ & -5.2 & -0.5 & 561.9 & $562.5 \pm 1.6$ \\
$\mathrm{Xe}(54)$ & -6.9 & -1.6 & 1028.1 & $1027.2 \pm 3.5$ \\
$\mathrm{Dy}(66)$ & -8.6 & -2.5 & 1336.6 & $1341.6 \pm 4.3$ \\
$\mathrm{~W}(74)$ & -9.4 & -3.4 & 1574.6 & $1568 \pm 15$ \\
$\mathrm{Bi}(83)$ & -11.0 & -4.5 & 1882.7 & $1876 \pm 14$ \\
\hline
\end{tabular}

Our results for the difference in the ionization potential between hydrogenlike and heliumlike ions are given in Table II. There is general agreement between our results and calculations using several different theoretical approaches. The results of one theory, a calculation by Lindgren et al. [12] using relativistic many body perturbation theory, are included in the table. The "2nd order many body" and "two-electron Lamb shift" contributions are tabulated separately so that they can be compared to our experimental uncertainties. The experimental uncertainties are smaller than the 2nd order many body contributions (except at higher Z), so the experimental results are sensitive to this part of the theory, but not yet to the two-electron Lamb shift. However, anticipated future improvements in the experimental precision could provide a significant test of the two-electron Lamb shift in heliumlike ions.

\section{Transition Energies In Few-Electron Ions}

Accurate calculations of the transition energies in high- $Z$ ions with more than two electrons require theoretical techniques different from those applied to heliumlike ions. Recent Super EBIT measurements of the $2 s_{1 / 2}=2 p_{3 / 2}$ transitions in highly charged uranium [13] and thorium [14] ions provide an ideal data set for testing our understanding of multielectron ions because the measurements span eight ionization stages (lithiumlike through neonlike) in both elements, so the effect of a varying number of electrons can be studied.

The Super EBIT measurements used a high resolution Bragg crystal of the von Hamos type, arranged as shown in Fig. 6 [15]. A typical X-ray spectrum from thorium ions is shown in Fig. 7. Several charge states are present in the trap simultaneously, resulting in multiple but well separated lines in the spectrum. The charge state distribution was shifted to cover the full range of interest by adjusting the Super EBIT operating parameters. The spectrometer was energy calibrated with the Lyman series of $\mathrm{x}$ rays in hydrogenlike and heliumlike argon, as their energies are well known. 


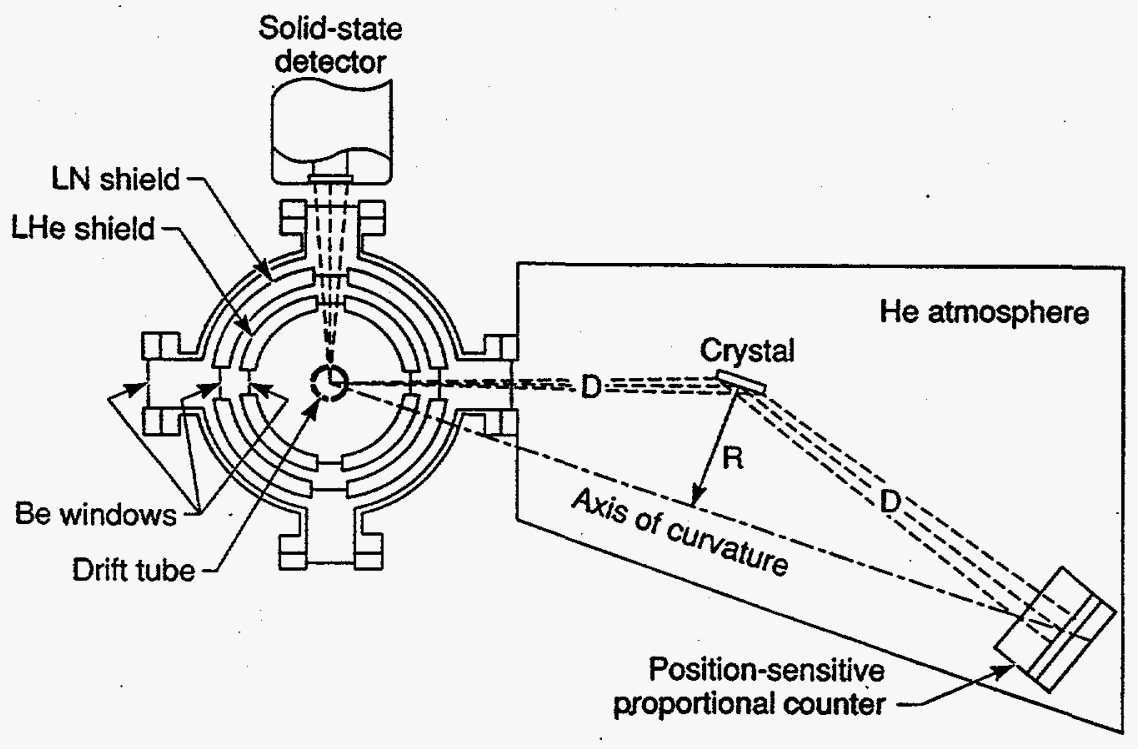

FIGURE 6. Diffraction plane layout of the high resolution von Hamos spectrometer used for the 2s-2p transition energy measurements. The electron beam direction is out of the page. The axis of curvature of the cylindrically bent crystal is the line joining the $x$-ray source and $x$-ray detector; the source-to-crystal and crystal-to-detector distances are equal. A solid state detector used for monitoring the EBIT $x$-ray emission is also shown.
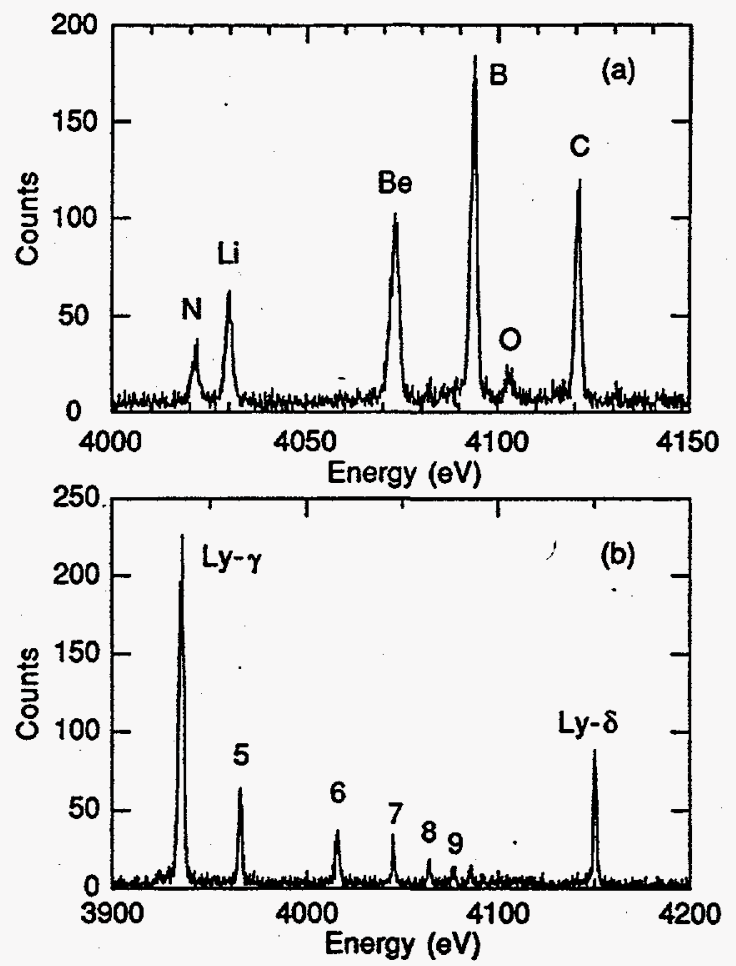

FIGURE 7. (a) The $2 s_{1 / 2}-2 p_{3 / 2}$ transition in lithiumlike $\mathrm{Th}^{87+}$ (labeled "Li") and other charge states of thorium excited at $100 \mathrm{keV}$ electron energy. (b) The Lyman series in hydrogenlike and heliumlike argon used for wavelength calibration. 


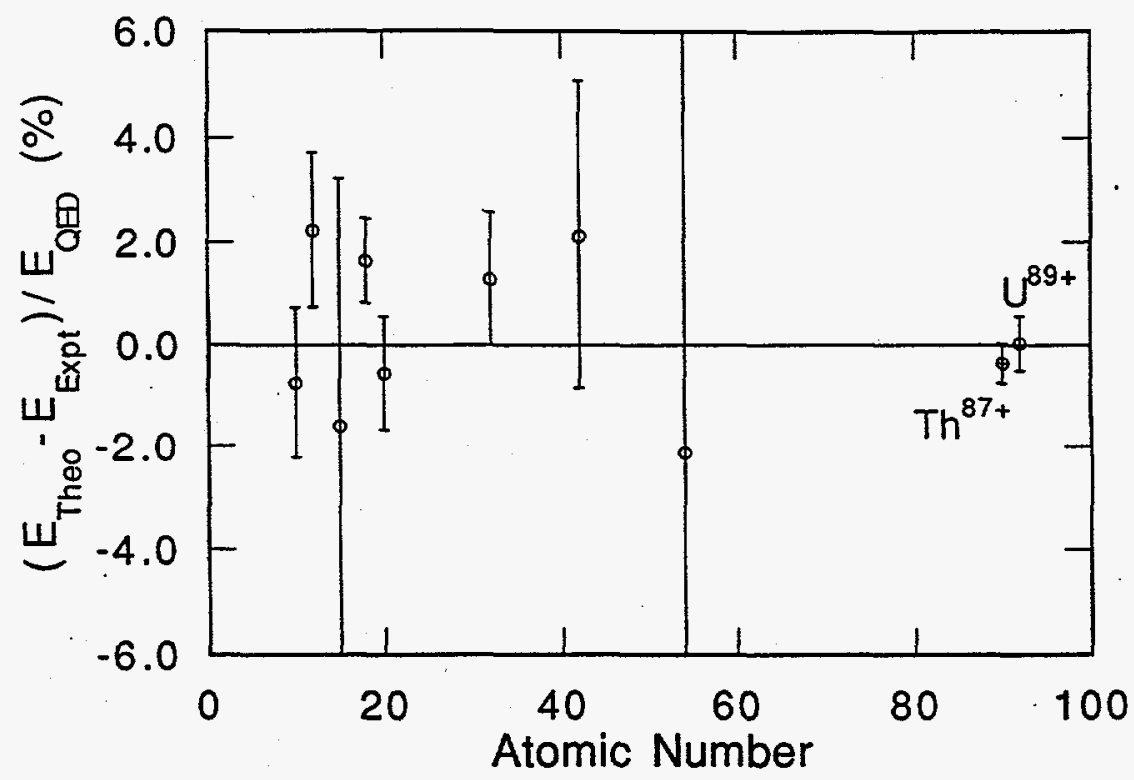

FIGURE 8. Difference between theory [16] and experiment for the $2 s_{1 / 2}-2 p_{3 / 2}$ transition in the lithiumlike isoelectronic sequence expressed as a percentage of the theoretical QED energy. The uranium and thorium points are from the Super EBIT at LLNL.

In Fig. 8 the measured energies for the $2 s_{1 / 2}-2 p_{3 / 2}$ transition in lithiumlike ions are compared to the theoretical QED energy [16] for this transition. Our Super EBIT results at high $Z$ provide the best test of theory. For high- $Z$ ions with several bound electrons, multiconfiguration Dirac-Fock (MCDF) theory has been a common tool for calculating energies. A comparison between MCDF theory and the Super EBIT results shows a systematic difference between theory and experiment that increases with the number of bound electrons $[13,14]$. This is attributed to the treatment of correlation energies in the MCDF calculations. More recently, notable progress has been made using many body perturbation theory [17] and configuration interaction techniques, resulting in better agreement with our Super EBIT measurements for the multielectron ions.

\section{NOVEL TECHNIQUES FOR SPECIAL MEASUREMENTS}

\section{Rare Isotopes And Nuclear Sizes}

Most EBIT and Super EBIT experiments have been done without regard to the isotopic composition of the element being studied, and large (gram) quantities of feed material with the natural isotopic abundance have been used for injection into the EBIT trap. However, several interesting experiments require isotopes or elements available only in trace quantities. Examples include ${ }^{233} U$, ${ }^{235} \mathrm{U}$, and ${ }^{249} \mathrm{Cf}$. These isotopes cannot be injected into Super EBIT with the usual 
MEVVA source [18] because the quantity of feed material required is too large. To solve this problem, we have developed a novel injection technique that enables Super EBIT operation with only nanogram quantities of feed material, 100-milliontimes smaller than previously possible [19].

The new technique works by mounting or plating a small amount of the desired rare isotope on the pointed end of a wire probe. As shown in Fig. 9, the wire probe is positioned near the compressed electron beam of our Super EBIT in the region where the beam passes through the ion-trap structure. The electron beam is surrounded by a cloud of trapped ions, some of which impact the sample and remove material from it by sputtering. On the order of $0.1 \%$ of the sputtered material comes off as positive ions and is captured in the EBIT trap. The rate of sputtering is controlled by the position of the probe and the bias voltage applied to it.

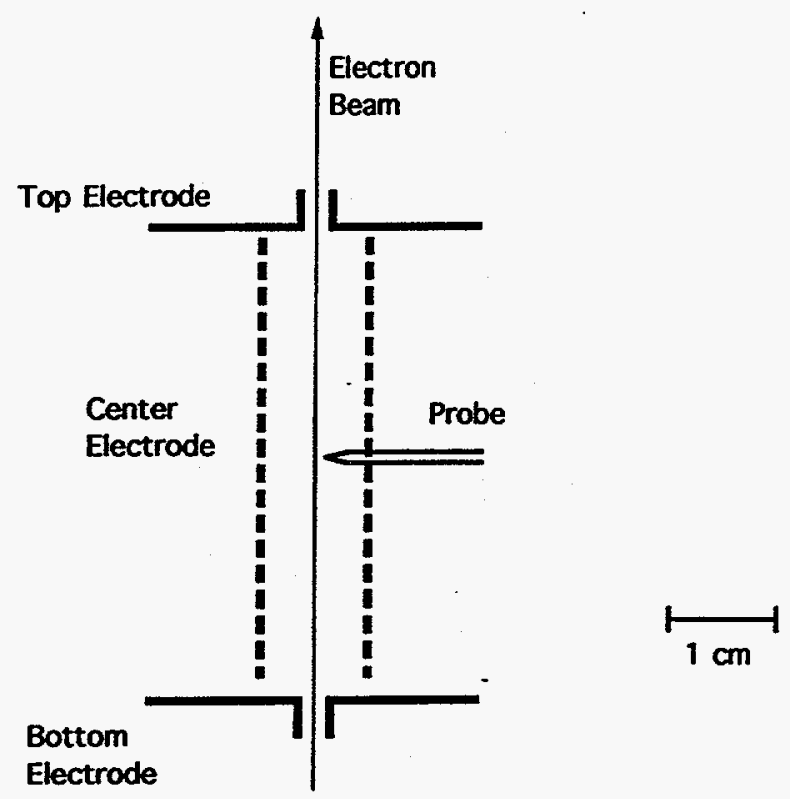

FIGURE 9. A schematic of the wire probe injector showing the placement of the probe with respect to the electron beam and trap electrodes. The probe location is controlled by a standard vacuum positioning device.

In one application of this new capability, we have measured the isotope shift of $\mathrm{x}$-ray transitions in high ionization stages of three uranium isotopes, ${ }^{233} \mathrm{U}$, ${ }^{235} \mathrm{U}$, and ${ }^{238} \mathrm{U}[20]$. The results provide information on the nuclear charge radius for the different isotopes. A high-resolution Bragg-crystal spectrometer was used to record the $2 s_{1 / 2}-2 p_{3 / 2}$ transitions in lithiumlike, berylliumlike, boronlike, and carbonlike uranium for the different isotopes. ${ }^{233} \mathrm{U}$ and ${ }^{235} \mathrm{U}$ were obtained from the rare isotope injector, and the more abundant ${ }^{238} \mathrm{U}$ was obtained from a MEVVA injector. 

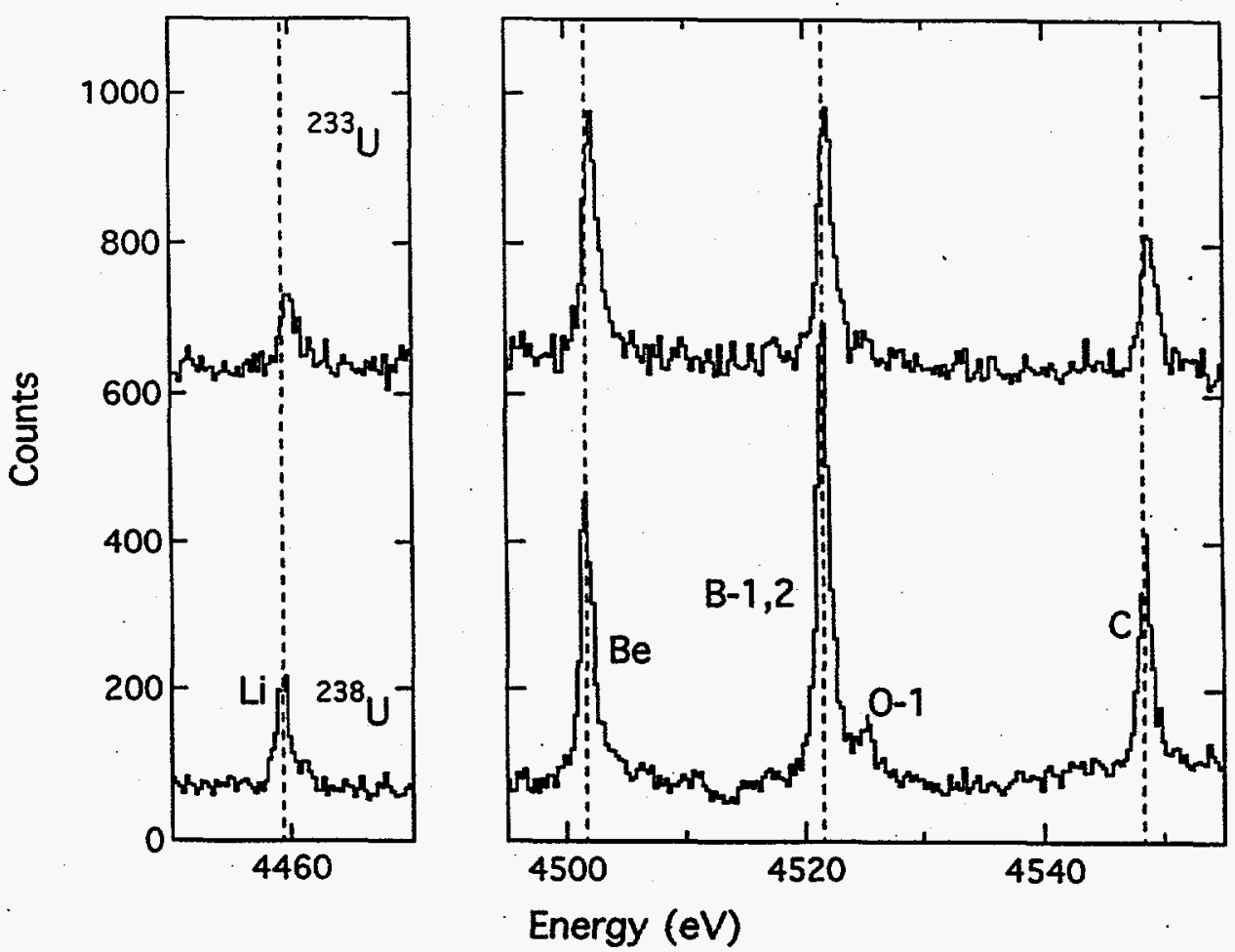

FIGURE 10. Comparison of $2 s_{1 / 2}-2 p_{32}$ transitions for ${ }^{233} U$ and ${ }^{238} U$. The $x$-ray lines are labeled with the chemical symbols for the different ionization stages.

A comparison of the spectra for ${ }^{233} \mathrm{U}$ and ${ }^{238} \mathrm{U}$ is shown in Fig. 10. The isotope shift of the transition energy can be clearly seen. The four different ionization stages give a consistent value of $\delta\left\langle r^{2}\right\rangle^{233,238}=-0.457 \pm 0.043 \mathrm{fm}^{2}$ for the difference in root-mean-square nuclear charge radius between ${ }^{233} \mathrm{U}$ and ${ }^{238} \mathrm{U}$. This may be compared with a previous value of $\delta\left\langle r^{2}\right\rangle^{233,238}=-0.383 \pm 0.044 \mathrm{fm}^{2}$ derived from measurements of $K \alpha$ and optical transitions in neutral uranium [21].

\section{Cold Ions And X-Ray Line Widths}

Observed $\mathrm{x}$-ray lines from radiative transitions in highly charged ions are broadened by both the natural width of the transition and by the motion of the emitting ions. In measurements with accelerator beams, Doppler broadening (in detectors subtending a finite angle) can be very large because of the high velocity of the emitting ions. X-ray lines from (stationary) plasma sources are broadened by the thermal motion of the emitting ions. Ions confined in Super EBIT also have a thermal motion due to ion heating by the electron beam. However, the evaporative cooling process, always used to keep highly charged ions from boiling out of the Super EBIT electron beam, can be exploited to cool the trapped ions to surprisingly low temperatures. In fact, thermal broadening can be reduced to the 
point where the natural width of some transitions can be measured in Super EBIT if very high resolution spectrometers are used [22].

Bragg Crystal spectrometers arranged as shown in Fig. 6 can achieve very high resolving power with EBIT if the distances between the electron beam, Bragg crystal, and detector are made large. In one experiment, resolving powers as high as $\lambda / \Delta \lambda \approx 22,000$ were obtained for trapped heliumlike titanium ions, allowing the line broadening due to the thermal motion of the ions to be clearly seen [22]. The $1 \mathrm{~s}^{2}\left({ }^{1} \mathrm{~S}_{0}\right)$ - $1 \mathrm{~s} 2 \mathrm{p}\left({ }^{3} \mathrm{P}_{2}\right)$ transition in heliumlike titanium was selected because the natural width of this forbidden line is too small to contribute to the observed line width.

The temperature of the trapped heliumlike titanium ions was reduced primarily by lowering the axial trapping voltage, an effect loosely analogous to lowering the temperature of boiling water by reducing the pressure. This cooling technique has been used in Super EBIT to cool heliumlike uranium ions to an estimated temperature of roughly $2 \mathrm{q} \mathrm{eV} \mathrm{[1],} \mathrm{suggesting} \mathrm{that} \mathrm{a} \mathrm{measurement} \mathrm{of} \mathrm{the}$ natural width may be possible for certain short-lived E1 decays in highly charged ions.

\section{CONCLUDING REMARKS}

The Super EBIT at LLNL supports an active and diverse research program with many notable accomplishments since the initial operation of Super EBIT in 1992. Recent work has focused on the few-electron ions of heavy elements, otherwise available only as high velocity beams from large accelerators. Looking to the future, it should be noted that the precision of many of the results reviewed above is limited by counting statistics; hence, future increases in x-ray intensity could substantially improve the experimental precision, probably to the point where even more subtle corrections to the structure of highly charged ions could be investigated. The variety of experiments will expand even further with the advent of Super EBIT devices under construction at other laboratories.

\section{ACKNOWLEDGMENTS}

Experiments at the LLNL Super EBIT were done in collaboration with $P$. Beiersdorfer, J. Crespo, S. Elliott, D. Knapp, D. Schneider, Th. Stöhlker, and K. Widmann. This work was performed under the auspices of the U. S. Department of Energy by Lawrence Livermore National Laboratory under Contract No. W7405-Eng-48.

\section{REFERENCES}

[1] R. E. Marrs, S. R. Elliott, and D. A. Knapp, Phys. Rev. Lett. 72, 4082 (1994). 
[2] D. A. Knapp, R. E. Marrs, S. R. Elliott, E. W. Magee, and R. Zasadzinski, Nucl. Instrum. Methods A 334, 305 (1993).

[3] J. H. Scofield, Phys. Rev. A 40, 3054 (1989)

[4] N. Claytor, B. Feinberg, H. Gould, C. E. Bemis, J. G. Campo, C. A. Ludemann, and C. R. Vane, Phys. Rev. Lett. 61, 2081 (1988).

[5] H. L. Zhang and D. H. Sampson, Phys. Rev. A 42, 5378 (1990); K. J. Reed (private communication).

[6] D. L. Moores and K. J. Reed, Phys. Rev. A 51, R9 (1995).

[7] C. J. Fontes, D. H. Sampson, and H. L. Zang, Phys. Rev. A 51, R12 (1995).

[8] D. A. Knapp, R. E. Marrs, M. A. Levine, C. L. Bennett, M. H. Chen, J. R. Henderson, M. B. Schneider, and J. H. Scofield, Phys. Rev. Lett. 62, 2104 (1989).

[9] D. A. Knapp, R. E. Marrs, M. B. Schneider, M. H. Chen, M. A. Levine, and P. Lee, Phys. Rev. A 47, 2039 (1993).

[10] D. A. Knapp, P. Beiersdorfer, M. H. Chen, J. H. Scofield, and D. Schneider, Phys. Rev. Lett. 74, 54 (1995).

[11] R. E. Marrs, S. R. Elliott, and Th. Stöhlker, Phys. Rev. A, in press.

[12] I. Lindgren, H. Persson, S. Salomonson, and P. Sunnergren, Proceedings of the Nobel Symposium on Trapped Charged Particles and Related Fundamental Physics, August, 1994 (to appear in Physica Scripta).

[13] P. Beiersdorfer, D. Knapp, R. E. Marrs, S. R. Elliott, and M. H. Chen, Phys. Rev. Lett. 71, 3939 (1993).

[14] P. Beiersdorfer, A. Osterheld, S. R. Elliott, M. H. Chen, D. Knapp, and K. Reed, unpublished.

[15] P. Beiersdorfer, R. E. Marrs, J. R. Henderson, D. A. Knapp, M. A. Levine, D. B. Platt, M. B. Schneider, D. A. Vogel, and K. L. Wong, Rev. Sci. Instrum. 61,2338 (1990).

[16] S. A. Blundell, Phys. Rev. A 47, 1790 (1993).

[17] W. R. Johnson, J. Sapirstein, and K. T. Cheng, Rev. A 51, 297 (1995).

[18] I. G. Brown, J. E. Galvin, R. A. MacGill, and R. T. Wright, Appl. Phys. Lett., 49, 1019 (1986).

[19] S. R. Elliott and R. E. Marrs, Nucl. Instrum. Methods B 100, 529 (1995).

[20] S. R. Elliott et al., unpublished.

[21] A. Anastassov, Yu. P. Gangrsky, K. P. Marinova, B. N. Markov, B. K. Kul'Djanov and S. G. Zemlyanoi, Hyperfine Interactions 74, 31 (1992).

[22] P. Beiersdorfer, V. Decaux, S. R. Elliott, K. Widmann, and K. Wong, Rev. Sci. Instrum. 66, 303 (1995). 